\title{
A compact and portable PC-based Gundestrup-Langmuir probe diagnostic system
}

\author{
P. Sicard, C. Boucher, A. Litnovsky, and J.-P. St-Germain \\ Université du Québec-INRS, Varennes, Québec J3X 1S2, Canada
}

(Received 27 February 2004; accepted 10 September 2004; published online 22 December 2004)

\begin{abstract}
A compact Gundestrup-Langmuir probe diagnostics system capable of data acquisition as well as data analysis was conceived at INRS-EMT, Canada, and used at IPP-FZJ, Germany. Data acquisition and analysis can be done with this system using several types of probes (Langmuir, double-Langmuir, Mach, Gundestrup,...). The versatility as to the different types of probe that one can use and the relative small size of the whole system makes it advantageous. Using a laptop computer makes the system small size and highly portable. The system acquires data at $1000 \mathrm{scans} / \mathrm{s}$ at 12 bit resolution on two probe systems simultaneously, using a total of 12 input channels. Bias is done by a DAC-ADC card and is amplified to give a $\pm 100 \mathrm{~V}$ sweeping range. Measured temperatures ranged from $0.75 \mathrm{eV}$ to $6.0 \mathrm{eV}$ with densities observed as low as 1 $\times 10^{10} \mathrm{~cm}^{-3}$ up to $5 \times 10^{11} \mathrm{~cm}^{-3}$, which are the range of conditions to be found in our experimental device. This system is also easy to reproduce since the hardware is commercially available and the scripts can be duplicated and modified according to the specifics of the hardware. (C) 2005 American Institute of Physics. [DOI: 10.1063/1.1834705]
\end{abstract}

\section{INTRODUCTION}

Electrical probes are a simple, effective, and widely used method to perform local measurements of plasma parameters. ${ }^{1,2}$ The particular design of the acquisition system described in the present paper enables us to use any type of electrical probe such as single Langmuir probes, multiple Langmuir probes, Mach probes or Gundestrup probes. ${ }^{3} \mathrm{We}$ have designed a compact system with computer-controlled acquisition that also includes the analysis software. Using a laptop computer renders the main component of the system portable. This acquisition system was designed to be used with a Gundestrup type probe mounted along with a double Langmuir probe to simultaneously perform plasma flow measurements and plasma characterization. ${ }^{4}$ The Gundestrup system consists of an array of collectors placed around the axis of the probe head at varying angles with the magnetic field covering $360^{\circ}$. Ion saturation currents $\left(I_{\text {sat }}\right)$ of opposing collectors are compared to deduce plasma flow velocities.

The performance of this system compares well with existing available commercial products. The acquisition speed is somewhat lower, which is due to the way the system is programmed, and will be covered in the appropriate section. The range of temperature and densities it can measure is comparable with what is available commercially. The feature that is lacking is a fully automated data analysis program. On the other hand, the specificity of this system is the ability to use a Gundestrup type probe, or any other type of multicollector probe that require many input channels. In our case, 12 input channels are used on 2 circuits running simultaneously and independently from one another.

The hardware components of the system will first be reviewed followed by the software components, both acquisition and analysis. Finally we will present a sample measured characteristic and its analysis.

\section{DIAGNOSTIC SYSTEM}

\section{A. Hardware components and principle of acquisition}

The compact probe diagnostic system consists of a DAC-ADC (Digital to Analog Converter-Analog to Digital Converter) card, a laptop computer, resistive divider with connectivity circuit and probe. The computer controlled DAC-ADC card first generates the biasing signal, which is amplified by a current/voltage amplifier to bias the electrical probes. The collected current is read by the card. The resistive divider is placed as an intermediate step between the signal amplifier, the probe and the card input. A schematic of the hardware components is shown in Fig. 1.

The DAC-ADC card is a National Instruments DAQcard 6024E for PCMCIA bus which is designed for portable computers. It has 2 analog output channels and 16 singleended input channels, or 8 differential input channels. The channels have a 12 bit resolution. The output channels are used to simultaneously bias two circuits attached to two sets of collectors that sweep different voltage range. The collected currents and the applied voltages are read via the input channels. The input signals are limited to a maximum of $\pm 10 \mathrm{~V}$. These limitations require the use of the resistive divider. The current-voltage $(I-V)$ characteristics of the Gundestrup and Langmuir collectors are measured over different voltage ranges requiring two independent output channels.

The choice of a PCMCIA card was made to enable the use of a laptop computer, making the main component of the acquisition system portable. The computer used is a Toshiba Satellite Pro 6100 portable personal computer using a Pentium 4 processor running at $1.4 \mathrm{GHz}$ with $256 \mathrm{Mb}$ of RAM. This enables control of the acquisition system with LABVIEW software, and also enables the analysis of the data using MAT- 


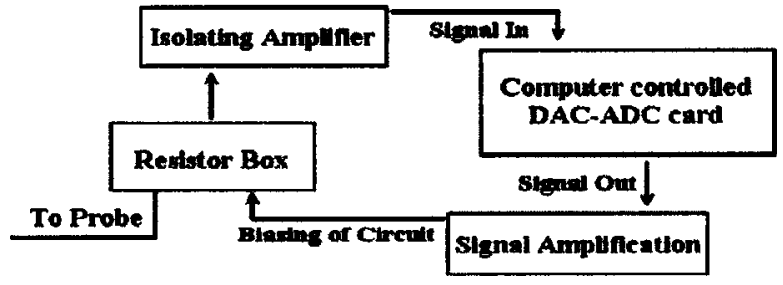

FIG. 1. Schematic of the computer-controlled acquisition system.

LAB scripts. These two last items will be described in the software section of this article.

The output signals coming from the card need to be amplified. Any type of operational amplifier will work. Using a Kepco BOP 200-1M operational amplifier that was configured to give a $10 \mathrm{~V} / \mathrm{V}$ amplification of the voltage, we are able to have a sweeping range of $\pm 100 \mathrm{~V}$. The amplification factor is the controlling parameter in determining the voltage range.

The currents collected by the probes are calculated from the voltage across a resistor in series with the probes. A circuit board was assembled consisting of 16 resistance channels. 8 of the channels have a 6-step position switch to select an appropriate value of the resistor on the circuit board. The resistances range from $0.5 \Omega$ to $50 \mathrm{k} \Omega$ by steps of one order of magnitude. This range was chosen to adapt to expected current values from our probes. This enables us to adjust to the range of the input signals which vary by four orders of magnitude, with a maximum at $200 \mathrm{~mA}$. The other 8 channels can operate in either of two operating modes: a current mode and a voltage mode. The current mode acts like the first 8 channels but with a fixed resistance. The voltage mode reads the voltage applied to the circuit. Out of the 16 channels available, 12 were used in two independent circuits. The first circuit was used for the Gundestrup probe using the first 9 channels, 8 reading the current and one monitoring the applied voltage. The second circuit was used for the Langmuir probes, again with one channel monitoring the voltage while the other collected the current. When using the probe in a double Langmuir configuration one less channel is

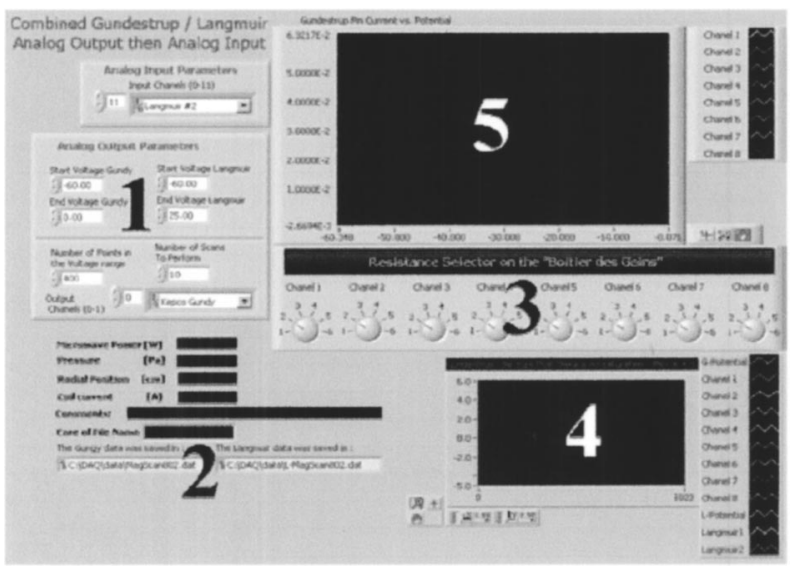

FIG. 2. View of the probe acquisition control panel. 1-Output parameters section. 2-Header information. 3-Resistances knobs. 4-Raw input monitor. 5-Measurement graph.

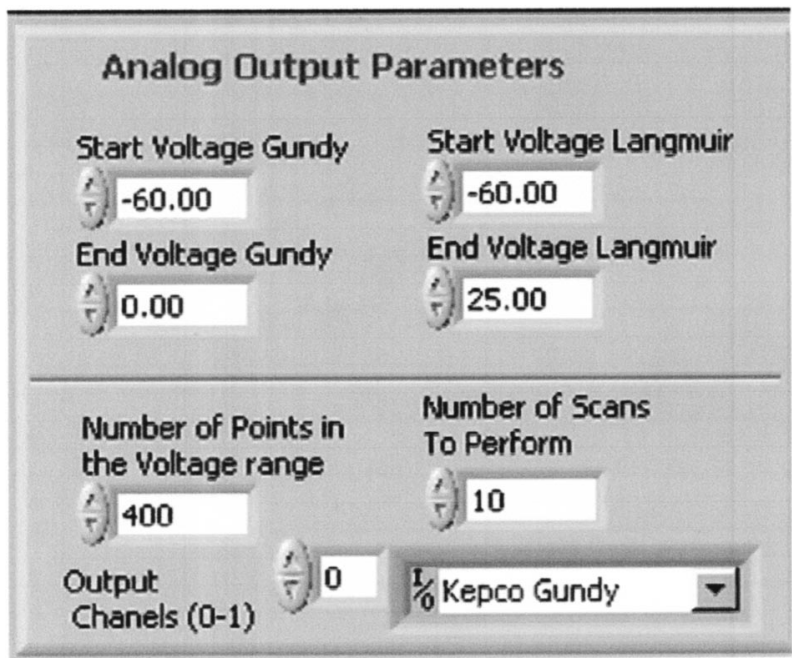

FIG. 3. View of entry fields to control the voltage range of the measurement.

needed. This whole circuit board is isolated and is mounted in a standard 19 in. rack-mount.

The voltages across each of the load resistors are measured by an Analog Devices 5B40 isolating-amplifier. These components amplify voltages by a factor 50 , having a maximum input of $100 \mathrm{mV}$, and a maximum output of $5 \mathrm{~V}$. We therefore have set the acquisition card to work in the $0-5 \mathrm{~V}$ range per channel.

\section{B. Software components \\ 1. Data acquisition}

The control of the acquisition system is done via software programmed on LABVIEW. The program starts with initialisation of the channels and then proceeds to the output/ input routine. After this routine, the raw input values are transformed to actual values of current and voltage, and are displayed on screen and stored on disk.

The user interface is designed as a control panel which is divided into five major sections which are shown in Fig. 2. The first (1) is the measurement parameters section where the user sets the voltage range and can select the acquisition channels to be used. In the header section (2) one fills in

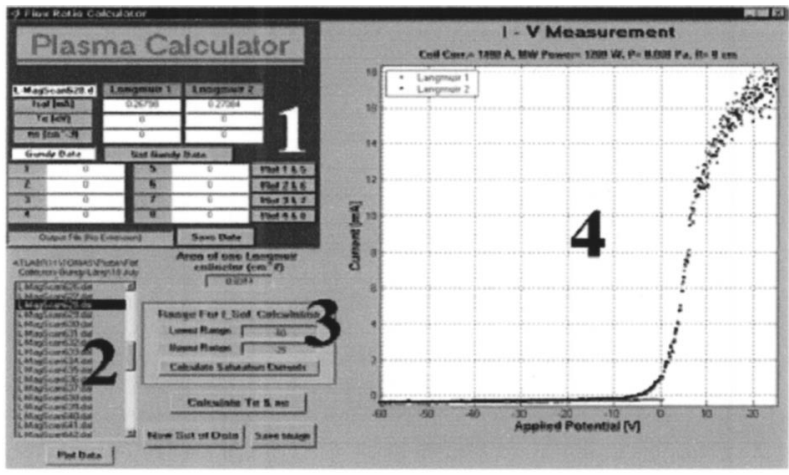

FIG. 4. View of the Graphical User Interface used to analyze the $I-V$ curves acquired via the acquisition system. 1-Fitted values obtained through analysis. 2-List of available data files. 3-Entry field for $I_{\text {sat }}$ fitting. 4-Visualization of $I-V$ curves. 


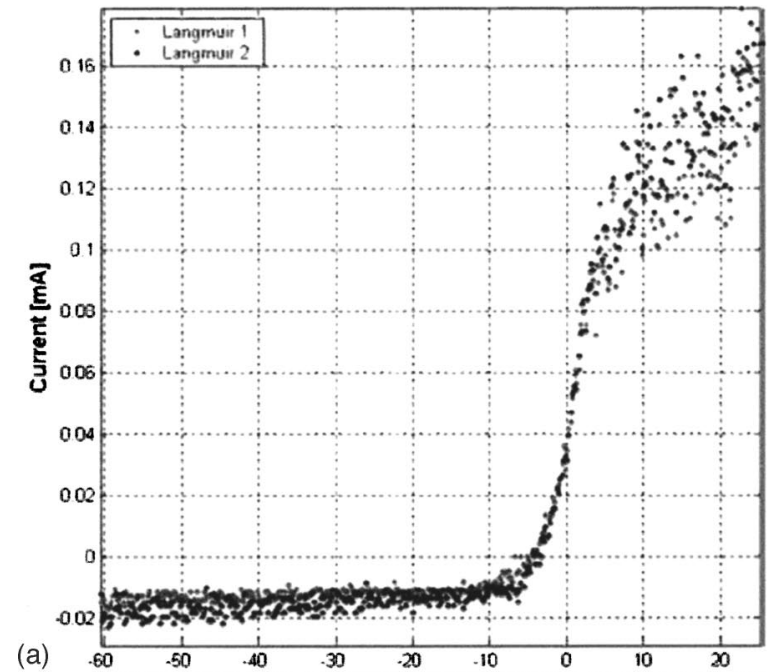

(a)
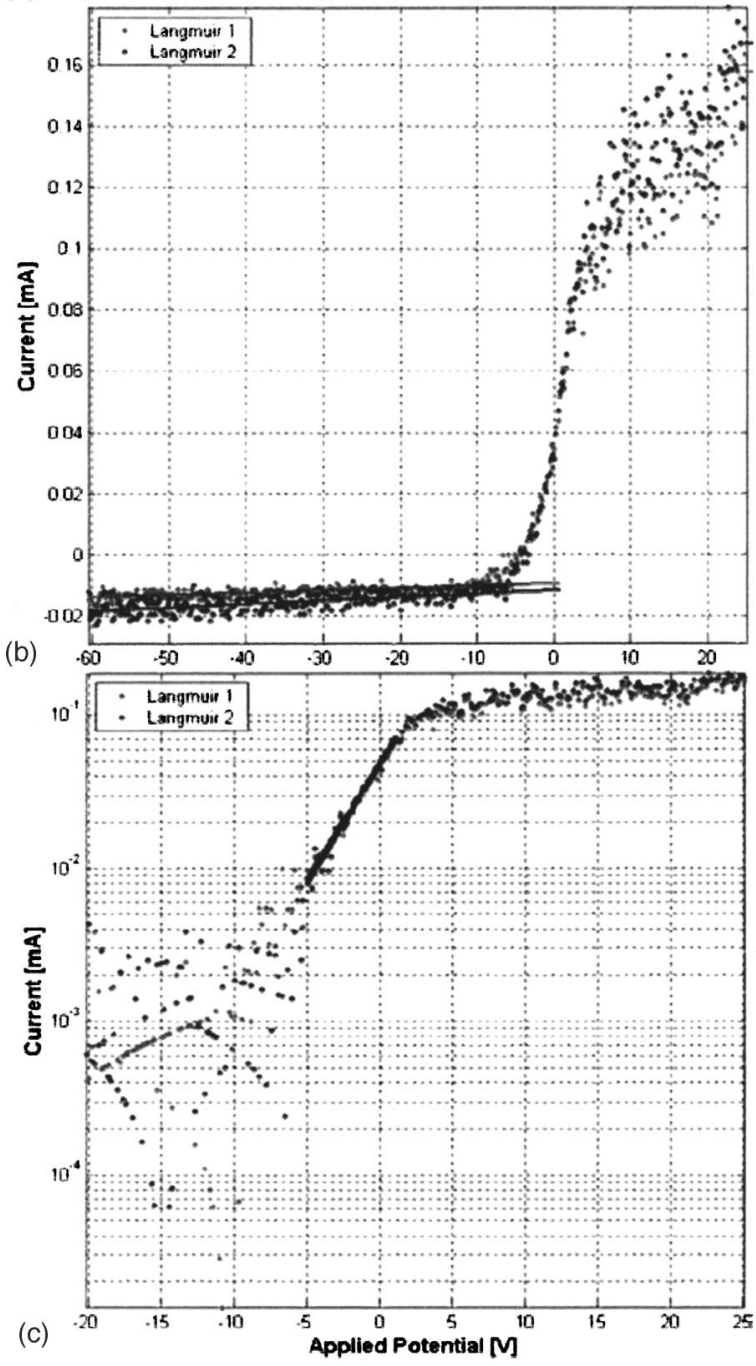

FIG. 5. Example of analysis of an $I-V$ characteristic. (a) Initial measurement. (b) Linear fit to deduce the $I_{\text {sat }}$ on each probe. (c) Reduced current characteristic where the linear fit was subtracted from the measured data and plotted on a semilog scale, where the fit to calculate $T_{e}$ can also be seen.

information that will be included in the data file such as measurement parameters. The third section (3) consists of a reproduction of the eight knobs that are used on the resistor box. The user must make sure that these knobs are at the same position than the switches on the resistance box before launching the routine to ensure correct data transformation. The raw input graph (4) is used to monitor the raw input to the acquisition card to avoid saturation of the channels which risk damaging the equipment. A graphic section (5) displays the measured characteristics and enables the user to see if the measurement is satisfactory or needs to be redone.

The voltage range for the $I-V$ curves is controlled by the user who sets the lower and upper voltages as well as the number of points to be digitized. This is limited to $\pm 100 \mathrm{~V}$ with our hardware configuration. The program sweeps the output voltage by incrementing by the appropriate $\Delta V$ to cover the whole voltage range. As can be seen from Fig. 3, the user can control the voltage range for the 2 probes independently. However, the number of points in the voltage range has to be the same. The last field, the Number of scans to perform, is included to get smoother characteristics. By repeating the measurements a certain number of times and averaging the readings, we can minimize the noise present in the measurements.

The output/input routine is a simple two-step sequence that sets the voltage on the output channels and then reads voltages on the input channels. The routine is repeated a number of times equal to the number of points in the voltage range that the user has specified. Finally, the whole range is repeated to get an averaged reading. The value of the output voltage is updated at every step of the routine. This use of the card to generate the biasing signal has the advantage to insure full control of the voltage applied to the probes at every step of the routine. However, this also limits the scanning speed to the output speed, since the later is usually always lower of the two on DAC-ADC cards. The acquisition speed is thus limited to $1000 \mathrm{scans} / \mathrm{s}$, which is the output rate of the PCMCIA bus card. To take advantage of the 200000 scans/s speed that the DAQ card offers, the acquisition script would have to be reprogrammed to include the addition of a signal generator. This would be done at the expense of software voltage control.

After each reading, raw values are accumulated in the buffer memory until the whole acquisition routine is completed. The raw input data are then transformed to take into account the resistors and amplifiers present on the circuit. The final $I-V$ characteristic is then saved to disk in ASCII format and is also shown on screen.

The header of the data file contains the operational parameters of the measurement such as neutral gas pressure, radial position of the probe, the time and date of the measurement as well as possible comments noted before launching the data acquisition. We use one file for the Langmuir probe data and one for the Gundestrup probe data due to geometrical considerations of the probe design, as well as practicality since the probes do not necessarily cover the same voltage range.

\section{Data analysis}

The analysis of the $I-V$ curves is done via a Graphical User Interface (GUI) that was programmed on MATLAB so that every function is activated by clicking the appropriate button. With this interface we can obtain the electron temperature $\left(T_{e}\right)$ and density $\left(n_{e}\right)$ of the plasma from the Lang- 


\begin{tabular}{|c|c|c|}
\hline L-MachRep118.d & Langmuir 1 & Langmuir 2 \\
\hline Isat $[\mathrm{mA}]$ & 0.00935 & 0.0116 \\
\hline Te $[\mathrm{eV}]$ & 2.97 & 2.76 \\
\hline$n e\left[\mathrm{~cm}^{\sim}-3\right]$ & $1.13 e+010$ & $1.45 e+010$ \\
\hline
\end{tabular}

FIG. 6. Values obtained after analysis of the data measured with the Langmuir probes. Also shown is the file name of the data being analyzed.

muir probes. In the case of the Gundestrup probe, we limit our analysis of the characteristics to the determination of the ion saturation currents $\left(I_{\text {sat }}\right)$ which are sufficient to deduce plasma flow. A screenshot of the GUI can be seen in Fig. 4. The different components will be outlined in this section.

We obtain the desired values via the relation for probe characteristic written in the form

$$
I_{p}=I_{\mathrm{sat}}+\Delta I V+I_{e} \exp \left[e V / T_{e}\right],
$$

where $T_{e}$ is in electron volts. The $\Delta I V$ term was introduces as a simplified approximation to account for the fact that the ion saturation current usually does not saturate. ${ }^{5}$

The first step in the analysis is the evaluation of the linear segment of the Langmuir probe characteristic, which is done by a linear fit. The user selects the measured data from the list of files located in a target folder and first plots the data. After visual inspection of the curve the user enters the voltage range to evaluate the linear segment of the characteristic. This contribution is then subtracted from the total current. The remaining current is the electron current onto the probe. It is plotted on a semilogarithmic scale so that the user can easily identify the exponential section of the curve. $T_{e}$ is then calculated via a fit of the slope of this section. With these values, $n_{e}$ is calculated via the relation

$$
I_{\mathrm{sat}}=0.61 n_{e} A_{\mathrm{eff}} c_{s},
$$

where $A_{\text {eff }}$ is the effective area of the probe and $c_{s}$ $=\left(T_{e} / m_{i}\right)^{1 / 2}$ is the ion sound speed for cold ions. Afterwards the user can proceed to analyse the Gundestrup data. Calculation of the $I_{\text {sat }}$ of each pin is done 2 collectors at a time to facilitate the visual inspection. All these calculated values are shown on screen and can be saved to a specified data file.

These fits used to calculate both $I_{\text {sat }}$ and $T_{e}$ are obtained by a pair-point averaging technique. From a vector of $n$ current elements, one directly calculates the slope between elements $i_{j}$ and $i_{j+n / 2}$, for all $n / 2$ pair of points. These slopes are then averaged to extract the mean slope of the set of points. This technique is simple to program and is more accurate to extrapolate a straight line out of a set of points than the usual least square fitting routine.

The linear segment of the data is determined by visual inspection of the user and the ranges of calculation are entered in the appropriate field. The result of the extrapolation is shown on the screen to see whether the result fits the data correctly. The range can be modified and the fit redone if the result is not satisfactory.

With the above technique, we were able to measure electronic densities $\left(n_{e}\right)$ ranging from $1 \times 10^{10} \mathrm{~cm}^{-3}$ up to 5 $\times 10^{11} \mathrm{~cm}^{-3}$ and electronic temperatures $\left(T_{e}\right)$ from $0.75 \mathrm{eV}$ to $6.0 \mathrm{eV}$, which are the expected values in our experimental device. Considering the range of current that the
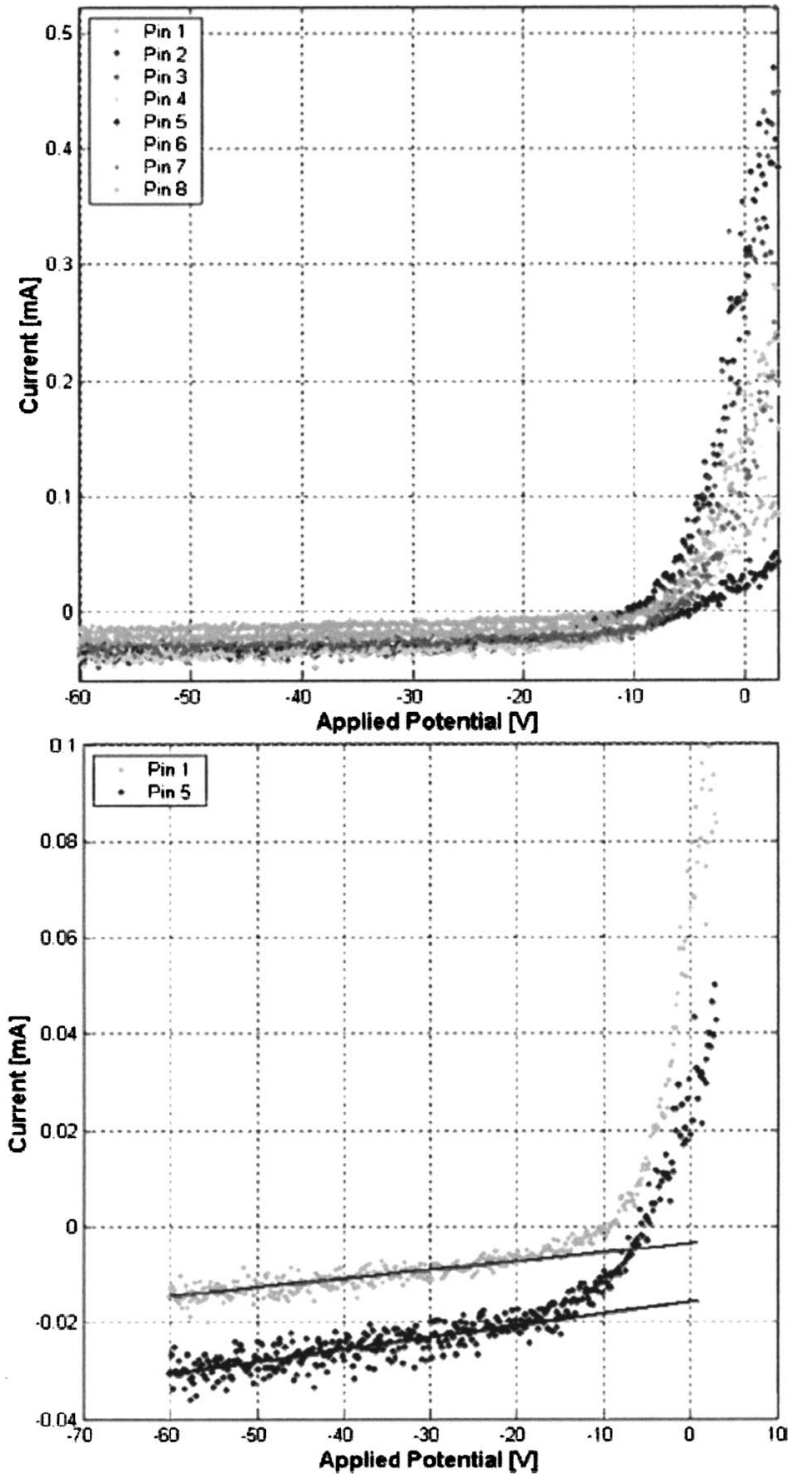

FIG. 7. Example of $I-V$ characteristics measured with an 8 collector Gundestrup probe. (a) All height curves are drawn to have an overview of the characteristics. (b) A pair of collectors is redrawn and a fit is plotted through the linear segment of the curve is shown.

circuit can handle and the programming of the system, it is expected that higher temperatures and densities could be measured.

The scripts for data acquisition and analysis are available to the community at http://claude.inrs-emt.uquebec.ca.

\section{SAMPLE MEASURED CHARACTERISTIC}

This system was successfully built, programmed and applied to acquire data at the TOMAS facility ${ }^{6}$ at the Institut für Plasmaphysik, Forschungszentrum Jülich (IPP-FZJ), Germany, for an argon plasma maintained by microwave radiation. This section will present the analysis of a sample measurement taken by this system.

A total of $10 I-V$ characteristics are taken by our probe to study one operational regime. The data taken with the Langmuir probes are used to determine the density and temperature while the data from the Gundestrup probe is used to determine the flow of the plasma. Figure 5 shows the graph- 
ics obtained in the different steps of the analysis of a sample measurement. Figure 5(a) shows the characteristics taken when functioning in a two single Langmuir probe mode. The characteristic has 400 points in a voltage range from $-60 \mathrm{~V}$ to $25 \mathrm{~V}$. Visual inspection of the measurement leads us to choose a range of $-60 \mathrm{~V}$ to $-20 \mathrm{~V}$ for the $I_{\text {sat }}$ calculation range, as it is the section that is linear. The fit obtained is shown in Fig. 5(b), which correlates well with the data.

When this first fit is found to be satisfactory, we can proceed to the evaluation of $T_{e}$. In Fig. 5(c) we can see the electron current plotted on a semilog scale, where the linear fit was subtracted from the total measured current. A linear section can be observed between $-5 \mathrm{~V}$ and $1 \mathrm{~V}$. These values were chosen as the range to fit $T_{e}$. Values of $2.97 \mathrm{eV}$ and $2.76 \mathrm{eV}$ are obtained for the probes, and are considered valid in view of the correlation of the fit as seen in the figure.

All the values deduced from the analysis can be seen in a section of the GUI that is presented in Fig. 6. From this the user has an immediate idea of the plasma parameters for a specific set of operational regime.

At the same position and in the same conditions, $8 \mathrm{I}-\mathrm{V}$ characteristics have been measured with the Gundestrup probe (Fig. 7). The voltage range is limited to a maximum of about $3 \mathrm{~V}$ since we are interested only in the $I_{\text {sat }}$ on each of these collectors. Analysis is done two curves at a time to facilitate visual inspection.

Fits obtained for a pair of opposite collectors are shown in Fig. 7(b) as an example. The range used to do the fit is from $-60 \mathrm{~V}$ to $-25 \mathrm{~V}$. Already on the graph we can see that the $I_{\text {sat }}$ ratio between the two collectors $\left(I_{\text {sat } 1} / I_{\text {sat5 }}\right)$ is different than unity. Since the pair of collectors analyzed are facing opposite directions, this ratio hints at the presence of a flow of plasma. The actual values of plasma velocity will be determined at a later stage with the proper models. ${ }^{7,8}$ However, this last analysis is beyond the scope of this paper.

${ }^{1}$ G. Matthews, Plasma Phys. Controlled Fusion 36, 1595 (1994).

${ }^{2}$ I. H. Hutchinson, in Principles of Plasma Diagnostics, 2nd ed. (Cambridge University Press, Cambridge, 2002), pp. 55-103.

${ }^{3}$ C. S. MacLatchy, C. Boucher, D. A. Poirier, and J. P. Gunn, Rev. Sci. Instrum. 63, 3293 (1992).

${ }^{4}$ A. Litnovsky, C. Boucher, P. Sicard, G. Lebrun, and J.-P. St-Germain, Czech. J. Phys. 53, 903 (2003).

${ }^{5}$ J. P. Gunn, C. Boucher, B. L. Stansfield, and S. Savoie, Rev. Sci. Instrum. 66, 154 (1995).

${ }^{6}$ H. B. Störk, J. Winter, J. Ihde, H. G. Esser, H. Reimer, and M. Freisinger, Fusion Technol. 39, 54 (2001).

${ }^{7}$ J. P. Gunn, Phys. Plasmas 8, 1995 (2001).

${ }^{8}$ H. Van Goubergen, R. R. Weynants, S. Jachmich, M. Van Schoor, G. Van Oost, and E. Desoppere, Plasma Phys. Controlled Fusion 41, L17 (1999). 